\title{
Approximation Techniques for Planar Periodic Structures
}

\author{
RICHARD C. COMPTON, STUDENT MEMBER, IEEE, AND DAVID B. RUTLEDGE, MEMBER, IEEE
}

\begin{abstract}
The rigorous calculation of electromagnetic properties of periodic meshes using moment methods requires considerable algebraic work and computer resources. In this paper, a number of easy to use approximation techniques for analyzing thin structures with square, rectangular, and circular holes are presented. Formulas for the effective impedance of these meshes are described which can easily take into account oblique incidence and the presence of a dielectric substrate. In addition, techniques for analyzing more complex-shaped apertures such as a cross are discussed. These methods are more accurate than existing approximation techniques and can be applied to a wide range of situations that could not be handled before.
\end{abstract}

\section{INTRODUCTION}

$\mathbf{P}$ ERIODIC MESHES are becoming increasingly important in the construction of microwave systems [1]-[4]. To design these systems efficiently, it is essential to be able to accurately predict mesh properties. The diffraction properties of meshes may be calculated very accurately using the method of moments [5]-[7] in which the electromagnetic fields are expanded in terms of Floquet and waveguide modes. Unfortunately, this method produces a relatively complicated set of equations which must be solved using a large computer. The difficulties involved with the rigorous moment method has led to the development of approximate methods for studying these meshes [8], [9].

A thin mesh may be modeled exactly by an impedance shunted across a transmission line. The equivalent-circuit model developed by Marcuvitz and later by Ulrich [2], [10], [11] was one of the first approximation methods for calculating the equivalent shunt impedance of singly and doubly periodic structures. This method is widely used because of its simplicity but has many limitations. For a mesh with square holes, the inductance is estimated by assuming its long wavelength behavior is like a strip grating whose inductance can be calculated using a conformal mapping [12]. The capacitance is chosen so that the free-space resonant wavelength is equal to the grid period. This approach has several limitations. The inductance and reso-

Manuscript received January 10, 1985; revised May 28, 1985. This work was supported in part by the Army Research Office and by the Jet Propulsion Laboratory.

The authors are with the Division of Engineering and Applied Science, California Institute of Technology, Pasadena, CA 91125.

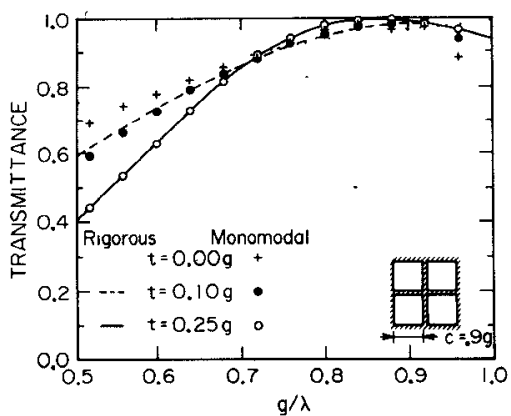

Fig. 1. Transmittance curves for normally incident radiation on a mesh with varying thicknesses. The mesh is characterized by a period $g$, thickness $t$, and a square hole size $c$. The monomodal calculations [3] make a good approximation to the rigorous calculations [7] for thicknesses $\geqslant 0.10 \mathrm{~g}$

nance estimate are both very inaccurate, particularly when the squares are small and a dielectric is present. In addition, the circuit model can only be applied to a small number of geometries-strips or square holes with $90^{\circ}$ periodicity axes at normal incidence. For thick meshes, good results have been obtained using methods based on the assumption that most of the energy is carried by one waveguide mode, all other modes being cut off [3]. For thin meshes, the monomodal approximation is good only at long wavelengths (see Fig. 1) because evanescent waveguide modes can carry energy through the mesh. If the hole shape is anything other than a rectangle, circle, or strip, moment methods that use waveguide modes as basis functions become complicated because the field can no longer be described in terms of simple waveguide modes [13]. The following sections present a number of approximation techniques for thin meshes which overcome the above limitations. The accuracy of the techniques will be displayed by comparing the results with rigorous solutions that are known to give excellent agreement with experiment [3].

\section{BACKGROUND THEORY}

Meshes may be classified as inductive or capacitive according to the long wavelength behavior of their equivalent shunt impedance. For the inductive and capacitive meshes of Fig. 2 at an air-dielectric interface (refractive index $n$ ), the equivalent circuit model of Ulrich predicts 


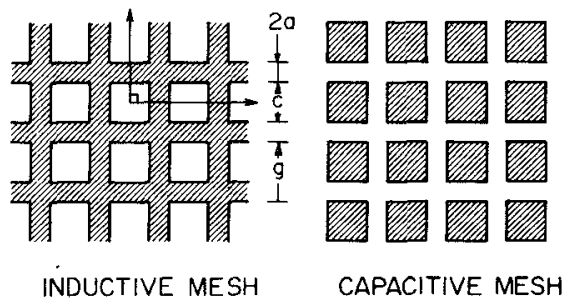

Fig. 2. Geometry of inductive and capacitive meshes with square holes and periodicity axes inclined at $90^{\circ}$

the following formula for energy transmittance [2], [12]:

$$
\begin{aligned}
T & =\frac{4 n}{(1+n)^{2}+\left(\frac{Z}{X}\right)^{2}} \\
\frac{X_{I}}{Z} & =-\left(\omega_{0} W\right)\left(\frac{\omega}{\omega_{0}}-\frac{\omega_{0}}{\omega}\right)^{-1} \\
\frac{X_{C}}{Z} & =\frac{2}{1+n^{2}}\left(4 \omega_{0} W\right)^{-1}\left(\frac{\omega}{\omega_{0}}-\frac{\omega_{0}}{\omega}\right) \\
W & =\ln \left(\operatorname{cosec} \frac{\pi a}{g}\right)
\end{aligned}
$$

where $\omega=g / \lambda$ is the normalized frequency and $\omega_{0}$ is the resonant frequency. The values $X_{I} / Z$ and $X_{C} / Z$ are the inductive and capacitive impedances normalized to the impedance of free space $Z$. At long wavelengths $(\omega \rightarrow 0)$, these impedances reduce to the strip grating impedances [12]

$$
\begin{aligned}
& \frac{X_{I}}{Z}=\omega W \\
& \frac{X_{C}}{Z}=\frac{-2}{1+n^{2}}(4 \omega W)^{-1} .
\end{aligned}
$$

Note that $X_{I} / Z$ is unaffected by the presence of a dielectric and $X_{C} / Z$ transforms like two capacitors in parallel.

For perfectly conducting meshes of infinitesimal thickness, the method of moments reduces to the following equations [5], [6]:

$$
Y^{M m} F_{m}=I^{M} \text {. }
$$

For the inductive mesh, the $F_{m}$ represent expansion coefficients for the electric field where

$$
\begin{aligned}
Y^{M m} & =\sum_{p q} \sum_{r=1}^{2}\left(\xi_{p q r}+y_{p q r}\right) C_{p q r}^{* M} C_{p q r}^{m} \\
I^{M} & =\sum_{r=1}^{2} A_{00 r} \xi_{00 r} C_{00 r}^{M} \\
C_{p q r}^{m} & =\iint_{\text {aperture }} \Phi_{p q r}^{*} \cdot \Psi^{m}
\end{aligned}
$$

and, for the capacitive mesh, the $F_{m}$ are coefficients in the current expansion with

$$
\begin{aligned}
Y^{M m} & =\sum_{p q} \sum_{r=1}^{2} \frac{1}{\left(\xi_{p q r}+y_{p q r}\right)} D_{p q r}^{* M} D_{p q r}^{m} \\
I^{m} & =\sum_{r=1}^{2} A_{00 r} \frac{\xi_{00 r}}{\left(\xi_{00 r}+y_{00 r}\right)} D_{00 r}^{m} \\
D_{p q r}^{m} & =\iint_{\text {aperture }} \Phi_{p q r}^{*} \cdot \Xi^{m} .
\end{aligned}
$$

The $\Phi_{p q r}$ are the Floquet modes, the $\Psi^{m}$ waveguide aperture modes, $\Xi^{m}$ are current modes and the $\xi_{p q r}$ and $y_{p q r}$ are the admittance of the Floquet modes on the two sides of the mesh [5], [6]. The $A_{00 r}$ are the coefficients of the incident field ( $r=1$ is TE and $r=2$ is TM). The solution of these equations involves the calculation of the integrals $C$ or $D$ and the inversion of matrix $Y$ whose elements are complex numbers. This requires considerable time and computer resources but can be made much easier by making a few simple approximations.

At wavelengths much longer than the grid period, the matrix $Y$ becomes dominated by the diagonal elements and the elements related to the primary mode ( $m=0$ say). Discarding all other terms results in a set of refined monomodal equations that may be solved analytically to yield the following expression for $F_{0}[5]$ :

$$
F_{0}=\frac{I^{0}-\sum_{\substack{m \neq 0 \\ m \neq 0}} \frac{Y^{0 m} I^{m}}{Y^{m m}}}{Y^{00}-\sum_{\substack{m \\ m \neq 0}} \frac{Y^{0 m} Y^{m 0}}{Y^{m m}}} .
$$

This may be used to calculate transmittance in a form which reduces to (1) and provides a considerably more accurate estimation of the long wavelength impedance than (3). For a TE incident wave, in the limit when the primary mode dominates, the terms in the sum of (8) are small and the impedance reduces to (2) with

$$
W=\frac{\pi\left|C_{001}^{0}\right|^{2}}{\sum_{p q}^{\prime}\left|C_{p q 1}^{0}\right|^{2} \sqrt{\alpha_{p}^{2}+\gamma_{p q}^{2}}} .
$$

The $\alpha_{p}$ and $\gamma_{p q}$ describe the spatial dependence of the Floquet modes [7], and the $\Sigma^{\prime}$ denotes the $p=0 q=0$ term is not summed. This equation provides a very general and accurate long wavelength mesh impedance for use with (1). In addition, this analysis can be used to make a good estimate of the frequency at which the mesh becomes resonant. Numerical studies show that at approximately the resonant frequency the matrix element $Y^{00}$ becomes real so that $X_{I} \propto W \propto 1 / \operatorname{Im}\left(Y^{00}\right)$ becomes infinite. Equation (9) expresses the long wavelength inductance in terms of a homogeneous, stationary function of the aperture field. The following section will illustrate the accuracy and versatility of this monomodal impedance method. 


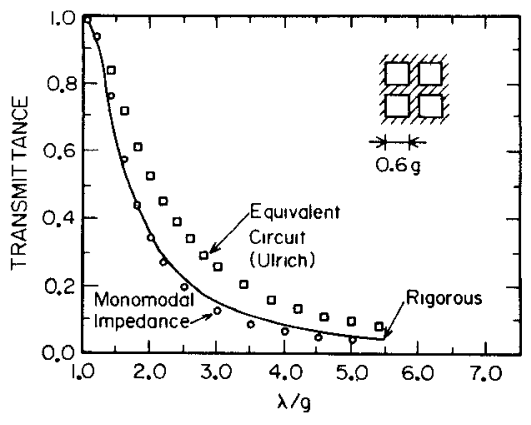

Fig. 3. Comparison at normal incidence of monomodal impedance and circuit model transmission curves with rigorous moment solutions.

\section{Calculations}

To check the approximation results, rigorous calculations [6], [7] were made using all waveguide modes up to $\mathrm{TE}_{55}$ and $\mathrm{TM}_{55}$. All Floquet modes up to $\sqrt{\alpha_{p}^{2}+\gamma_{p q}^{2}} \leqslant$ $11(2 \pi / g)$ were included. The addition of more modes made no significant change in the transmittance values. The results were checked using conservation of energy, Babinet's principle, and reciprocity and are estimated to be accurate to better than \pm 0.02 in the transmittance. When analyzing meshes with narrow metal strips $(c / g \geqslant 0.9)$, it was found that a much larger number of TE/TM modes were required to simulate the singularities at the metal edges.

Fig. 3 demonstrates the monomodal impedance formula (9) for a freestanding mesh with square holes. The fundamental propagating mode in the aperture is the $\mathrm{TE}_{10}$ mode. The sum over Floquet modes in ( 9 ) converges rapidly. For the results presented, $p$ and $q$ are summed from -11 to +11 . The monomodal formula gives a better estimation of impedance than Ulrich's circuit model [1], [2] and provides better overall transmittance predictions. Furthermore, as the strips become wider, Ulrich's equivalent-circuit model becomes more inaccurate because the circuit impedance estimate gets worse and the resonance occurs at longer wavelengths further from the grid period. On the other hand, the monomodal impedance estimation (9) becomes better, and the resonant frequency can always be accurately calculated. The monomodal impedance method allows the accurate calculations of transmission curves without large mainframes and long run times. Typical run times on a VAX 11-750 are $60 \mathrm{~s}$ for a complete transmission curve.

The transmission curve of Fig. 4 is for a mesh with periodicity axes inclined at $45^{\circ}$. Such a structure cannot be analyzed with Ulrich's equivalent-circuit theory. The refined monomodal treatment gives an excellent impedance value and a good estimate of the resonant frequency $\left(Y^{00}\right.$ purely real). Fig. 5 shows the same mesh for TE radiation incident at $45^{\circ}$-oblique incidence is another situation that could not be properly accounted for with existing circuit theory. At oblique incidence, the transmission formula (1) is modified since the impedance of a TE wave changes from $Z$ to $Z / \cos (\theta)$, where $\theta$ is the angle that the

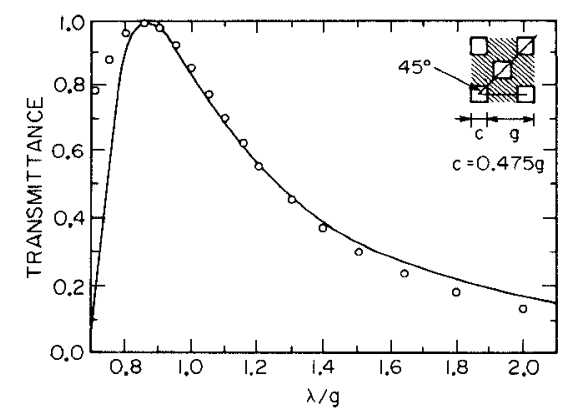

Fig. 4. Transmittance curve for a square mesh at normal incidence with periodicity axes inclined at $45^{\circ}$ comparing the rigorous solution (solid line) to the monomodal impedance approach $(\circ)$.

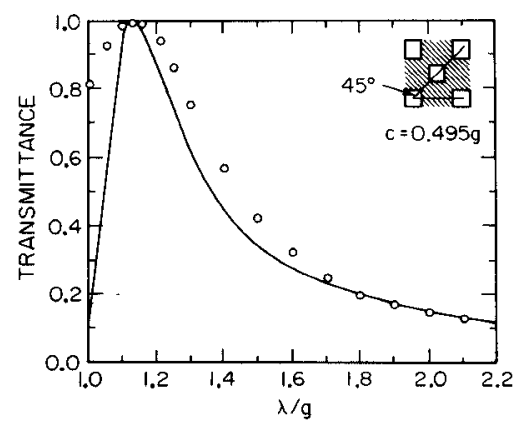

Fig. 5. Transmittance curve for a square mesh with periodicity axes inclined at $45^{\circ}$ and radiation incident at $45^{\circ}$ comparing the rigorous solution (solid line) to the monomodal impedance approach $(\circ)$.

magnetic field makes to the plane of the mesh. If $\theta_{t}$ and $\theta_{t}$ are the incident and transmitted angles then (1) becomes

$$
T=\frac{4 n \cos \left(\theta_{t}\right) \cos \left(\theta_{t}\right)}{\left[\cos \left(\theta_{t}\right)+n \cos \left(\theta_{t}\right)\right]^{2}+\left(\frac{Z}{X}\right)^{2}} .
$$

- A similar formula for a TM incident wave may be derived by using $Z \cdot \cos (\theta)$ for the transmission-line impedance.

The monomodal impedance method can also take into account the presence of a dielectric. Fig. 6 shows the transmittance curve for a mesh at a dielectric interface. The long wavelength inductive mesh impedance is unchanged. The dielectric alters the characteristic impedance on the transmission side of the structure and shifts the resonant wavelength. By modifying the transmission-line impedance, it is also a simple matter to consider a dielectric slab [5] and allow for absorption loss in the dielectric. This improved impedance formula can also be applied to circular holes or to any shaped hole or metal plate where the primary energy transmission mode can be calculated. If a more accurate estimation of the impedance is required then more terms in the sum of (8) may be included.

The refined monomodal formula becomes inaccurate when the aperture field or sheet current cannot be expressed in terms of a single mode. The method is limited to wavelengths greater than the resonant frequency and small angles of incidence $\left(\theta \leqslant 45^{\circ}\right)$. For the rectangular mesh with narrow strips $(c / g>0.9)$, the assumption that the 


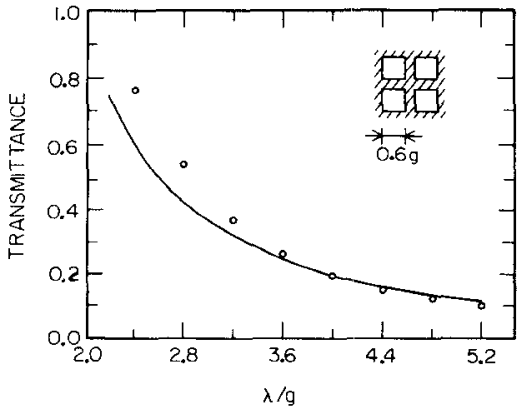

Fig. 6. Transmittance curve for a square mesh at normal incidence at an air dielectric boundary $(n=2.1)$ comparing the rigorous solution (solid line) to the monomodal impedance approach (o).

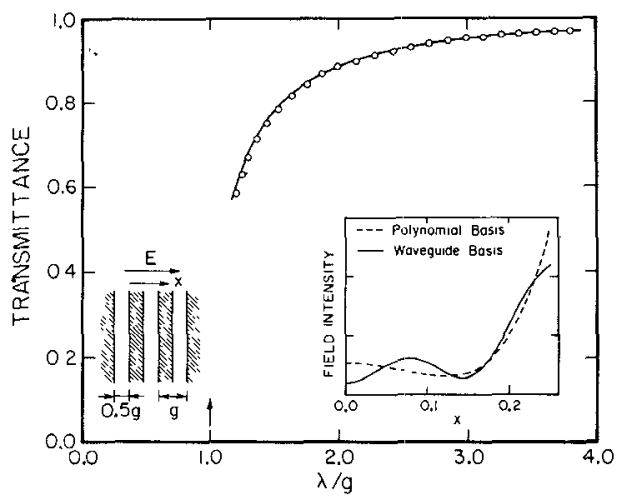

Fig. 7. Transmission curve for a strip gratıng comparıng waveguide basis approach (solid line) with a polynomial basis method $\left({ }^{\circ}\right)$.

$\mathrm{TE}_{10}$ is the only mode becomes less accurate than using (1) and (2).

\section{Gentral-Shaped Aperture}

When the shape of the aperture becomes anything other than a strip, square, or a circle, it becomes extremely difficult to find the waveguide or current basis functions $\Psi$ and $\Xi$ used to express the electric field or current. For infinitesimally thin meshes, there is no need to use these modes. Any set of independent functions that are continuous over the aperture and satisfy the boundary conditions on the aperture walls should be acceptable. This section will examine the use of some very simple functions for use in a wide range of mesh structures.

One of the simplest set of possible basis functions is the set of delta functions $\delta\left(x-x_{n}\right)$ where the $x_{n}$ are points in the aperture. The method of moments then reduces to least-square matching of the aperture field to the Floquet field above the mesh. Solutions obtained using delta functions are very sensitive to the positioning of the points $x_{n}$. In addition, accurate solutions require very large numbers of points and thus a very large matrix (5) needs to be inverted. The major advantages of using delta functions is that they can be easily used for any general-shaped aperture.

An examination of a typical aperture field obtained using traditional waveguide basis for a strip grating (Fig. 7 inset) shows that the field may be approximated by a

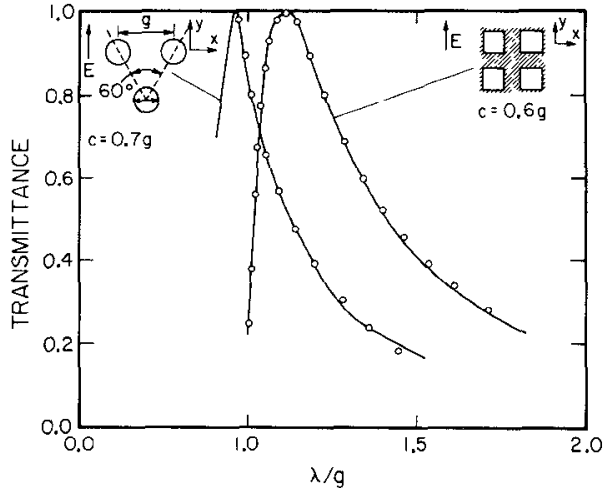

Fig. 8. Transmission curve for meshes with square and circular holes [14] comparing waveguide basis approach (solid line) with a polynomial basis method $(\circ)$.

simple polynomial expansion. The size of the matrix to be inverted is equal to the number of basis functions required to expand the field. It is important to exploit all symmetry properties of the structure under consideration because each symmetry reduces the size of the matrix by a factor of two. For the configuration of Fig. 7, the electric field in the aperture may be expanded as

$$
E_{x}=\sum_{n} c_{n} x^{n}
$$

where symmetry (for $E_{\text {incident }}$ parallel to the $x$-axis) requires that $n$ be even. Fig. 7 shows that such a simple expression for the aperture field, together with (5) and (6), can produce extremely accurate results for the transmittance even though the field differs slightly from the field predicted using waveguide modes.

Polynomial expansions may also be used with great success in doubly periodic meshes. In the case of a mesh with square holes (Fig. 8), the following expression for the two electric-field components may be used:

$$
\begin{aligned}
& E_{x}=\sum_{m n} C_{m n} g_{m n} x^{2 n+1} y^{2 m+1}\left(y^{2}-\frac{c}{2}\right) \\
& E_{y}=\sum_{m n} D_{m n} g_{m n} x^{2 n} y^{2 m}\left(x^{2}-\frac{c}{2}\right) .
\end{aligned}
$$

The allowed powers of $x$ and $y$ are determined from the symmetry and the orientation of the incident $E$ field (parallel to the $y$-axis). The factor $g_{m n}$ is chosen so that the integral of the square of the basis functions is normalized. If the functions are not normalized, the matrix (5) can become unstable because of large variations in the magnitude of its elements. The factors in brackets assure that the tangential field goes to zero on the walls of the aperture at $x= \pm c / 2$ and $y= \pm c / 2$. Fig. 8 shows that this polynomial expansion gives excellent agreement with the results predicted using the square waveguide modes.

For meshes with circular holes, the radial dependence may be expressed in powers of $r$ instead of the waveguide mode Bessel functions. The allowed angular dependence is chosen to take advantage of the $x-y$ symmetry. The integrals required to fill the matrix (eqs. (5)-(7)) may be 
evaluated numerically using a modified Simpsons rule integration over the angular and radial variables. Fig. 8 demonstrates the accuracy of this expansion method for circular holes placed in an equilateral array. If the structure consists of metal plates instead of holes, then similar expansions may be used to describe the current in the plates.

$\therefore$ - There are a number of numerical checks that can be carried out to verify the solutions. Firstly, conservation of energy requires that the energy carried away from the mesh equals the energy incident. In this formulation, energy conservation is an analytical result but is a good check of the computer implementation. Another check, applicable to square meshes, is the common phase properties of the $C_{m n}, D_{m n}$ and in particular [15]

$$
\text { Transmittance }=\sin ^{2}\left(\arg \left(C_{m n}\right)\right) .
$$

This provides a quick method of finding the transmittance without having to completely solve the matrix equation and reconstruct the Floquet fields. The solution may also be checked by comparing the aperture fields with the Floquet fields at the plane of the mesh. The numerical analysis of (6) suggests that continuity of the electric field is not necessarily a strong test of the solution but continuity of the normal magnetic field is a good measure of completeness of the aperture basis functions. Completeness of the Floquet modes can be checked with the following identity [13]:

$$
\sum_{p q r} C_{p q r}^{m} C_{p q r}^{* M}=\iint_{\text {aperture }} \Psi^{m} \cdot \Psi^{M}
$$

This equation is an extension of the result obtained for use with waveguide basis functions but does not require that the basis functions be orthogonal or normalized over the aperture. The identity is exact for an infinite number of independent basis functions and Floquet modes and holds approximately when both series are truncated. Numerical stability may be checked by looking at the determinant of the matrix (5). This is an easy step to do as part of the matrix inversion. When the solution is unstable, the determinant varies rapidly and the phase changes by $180^{\circ}$.

When the shape of the hole or plate becomes complicated, it is no longer possible to find simple functions defined over the entire aperture, which are continuous and satisfy the boundary conditions. If the aperture basis functions are not continuous over the aperture, the resulting discontinuity leads to spurious results. This problem can be avoided by splitting the aperture into smaller regions and requiring the functions defined over each region to vanish on the boundaries. If the boundary of the region coincides with the aperture wall, then only the tangential electric field or the normal current must vanish.

To illustrate this technique, consider the cross-shaped aperture. The aperture is split into a number of smaller overlapping regions and the field is expanded in terms of two-dimensional triangle functions. Fig. 9 shows good results using only a small number of functions. More accurate results can be obtained by using a larger number of smaller regions.

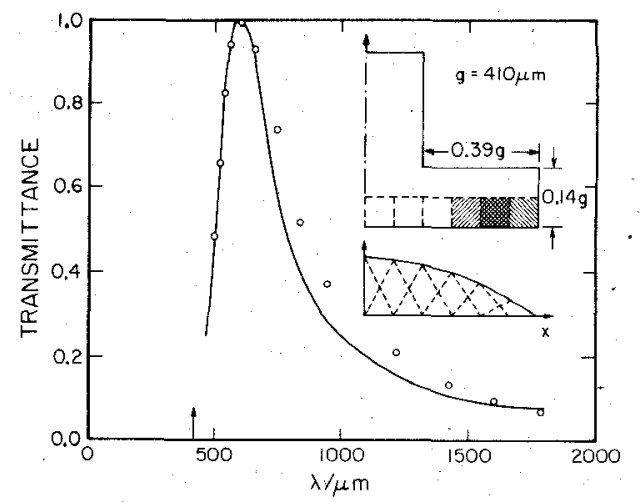

Fig. 9. Transmission curve for a meshes with cross-shaped holes comparing waveguide basis approach (solid line) with a two dimensional triangular basis method $\left({ }^{\circ}\right)$.

This approach may be used to solve a large number of boundary problems that have not been previously analyzed. Unlike other methods, it makes no assumptions about the form of the fields or currents and places no restrictions on the size or shape of the hole. For example, some techniques for analyzing loaded slots and crossed dipoles make restricted assumptions about the currents or electric fields present based on the stipulation that the aperture or plate is narrow along one of its transverse directions [16], [17].

The time required to obtain the transmittance at a given wavelength depends on the number of aperture modes, which determines the number of linear equations needing to be solved. Very quick run times can be achieved by choosing a small number of good basis functions. In general, run times are considerably shorter than for the rigorous method using waveguide modes.

In the method of moments [18], the unknown field is expanded in terms of a complete set of basis functions, and the resulting equation is projected onto a set of trail functions. Little work has been done on examining what constitutes a good set of trial functions and what restrictions need to be placed on the basis functions. In the above analysis, the trial functions and basis functions are the same (Rayleigh-Ritz method). In certain applications, it may be an advantage to use trial functions that differ from the basis functions. For example, in the case of the cross, step functions could be used as trial functions instead of triangle functions.

\section{CONCLUSIONS}

The first part of this paper presents a simple technique for calculating the effective impedance of a thin periodic mesh. This refined monomodal impedance technique is considerably faster and easier to use than rigorous moment methods and more accurate and versatile than existing approximation methods.

The later sections describe a number of ways of choosing nonwaveguide basis functions for the current or electric field. These functions can be used to solve problems like the strip grating, square hole mesh, and the circular hole mesh. They can also be extended to solve mesh problems 
like the cross in which the waveguide modes are not readily available.

\section{ACKNOWLEDGMENT}

The authors would like to thank R. C. McPhedran for his contributions to the finite thickness mesh calculations of Fig. 1. and for his help with the refined monomodal theory.

\section{REFERENCES}

[1] M. S. Durschlag and T. A. DeTemple, "Far-IR optical properties of freestanding and dielectrically backed metal meshes," in Appl. Opt., vol. 20, pp. 1245-1253, Apr. 1981.

. [2] R. Ulrich, "Interference filters for the far infrared," Appl. Opt., vol. 7, pp. $1987-1995$, Oct. 1968.

[3] R. C. McPhedran and D. Maystre, "On the theory and solar application of inductive grids," Appl. Phys., vol. 14, pp. 1-20, Jan. 1977.

[4] R. C. Compton, J. C. Macfarlane, L. B. Whitbourn, M. M. Blanco, and R. C. McPhedran, "Babinets principle applied to ideal beamsplitters for millimeter waves," Optica Acta, vol. 31, pp. 515-524, May 1984.

[5] C. C. Chen, "Transmission through a conducting screen perforated periodically with apertures," IEEE Trans. Microwave Theory Tech., vol. MTT-18, pp. 627-632, Sept. 1970.

[6] C. C. Chen, "Scattering by a two-dimensional periodic array of conducting plates," IEEE Trans. Antennas Propagat, vol. AP-18, pp. 660-665, Sept. 1970.

[7] R. C. McPhedran, G. H. Derrick, and L. C. Botten, Electromagnetic Theory of Gratings. Berlin: Springer 1980, ch. 7, pp. 227-276.

[8] R. Ulrich, "Far infrared properties of metallic mesh and its complementary structure," Infrared Phys., vol. 7, pp. 37-55, Jan. 1967.

[9] R. C. Compton, L. B. Whitbourn, and R. C. McPhedran, "Simple formula for the transmittance of strip gratings," Int. J. Infrared and Millimeter Waves, vol. 4, pp. 901-912, Nov. 1983

[10] N. Marcuvitz, Waveguide Handbook (MIT Radiation Laboratory Series, vol. 10). New York: McGraw Hill, 1951.

[11] R. C. Compton, L. B. Whitbourn, and R. C. McPhedran, "Strip gratings at a dielectric interface and applications of Babinets principle," Appl. Opt., vol. 23, pp. 3236-3242, Sept. 1984.

[12] L. B. Whitbourn and R. C. Compton, "Equivalent circuit formulae for metal grid reflectors at a dielectric boundary," Appl. Opt., vol. 24, pp. 217-220, Jan. 1985 .

[13] R. C. Compton, R. C. McPhedran, G. H. Derrick, and L. C. Botten, "Diffraction properties of a bandpass grid," Infrared Phys., vol. 23, pp. 239-245, May 1983.

[14] C. C. Chen, "Diffraction of electromagnetic waves by a conducting screen perforated periodically with circular holes," IEEE Trans. Microwave Theory Tech., vol. MTT-19, pp. 475-481, May 1971.

[15] L. C. Botten, R. C. McPhedran, and J. M. Lamarre, "Inductive grids in the resonant region: Theory and experiment," Submitted to Int. J. Infrared and Millimeter Waves.

[16] R. J. Luebers and B. A. Munk, "Cross polarization losses in periodic arrays of loaded slots," IEEE Trans. Antennas Propagat., vol. AP-23, pp. 159-164, Mar. 1975.
[17] C. Tsao and R. Mittra, "Spectral-domain analysis of frequency selective surfaces comprised of periodic arrays of cross dipoles and Jerusalem crosses," IEEE Trans. Antennas Propagat., vol. AP-32, pp. 478-486, May 1984.

[18] R. F. Harrington, Field Computation by Moment Methods. New York: Macmillan, 1968.

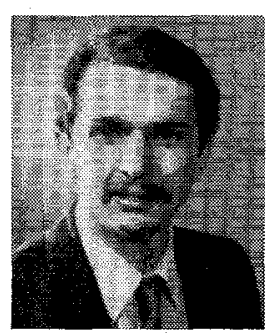

Richard C. Compton (S'84) was born in Sydney, Australia, on December 6, 1960. He completed his B.S. degree at the University of Sydney in 1982 and was awarded the University Medal in Physics.

In 1982-1983, he was associated with the Commonwealth Scientific and Industrial Research Organization, Division of Applied Physics, in Sydney, where he worked with the millimeter/submillimeter-wave group. He is now a Fullbright Scholar, pursuing his Ph.D. at the California Institute of Technology. He is currently working on the design of millimeter-submillimeter-wave arrays.

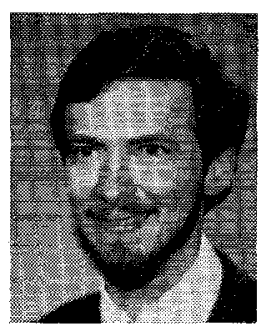

David B. Rutledge (S'75-M'80) was born in Savannah, GA, on January 12,1952 . He received the B.A. degree in mathematics from Williams College, Williamstown, MA, in 1973, the M.A. degree in electrical engineering from Cambridge University, Cambridge, England, 1975, and the $\mathrm{Ph} . \mathrm{D}$. degree in electrical engineering from the University of California at Berkeley in 1980.

In 1980, he joined the faculty at the California Institute of Technology, Pasadena, CA, where he is now Associate Professor of Electrical Engineering. He received the IBM Faculty Development Award and the NSF Presidential Young Investigator Award. His research is in developing millimeter- and submillimeter-wave monolithic integrated circuits and applications. 